\title{
A novel dual PI3K $\alpha / m$ TOR inhibitor PI-103 with high antitumor activity in non-small cell lung cancer cells
}

\author{
ZU-QUAN ZOU ${ }^{1 *}$, XIAO-HONG ZHANG ${ }^{2 *}$, FENG WANG $^{3}$, QI-JUN SHEN ${ }^{2}$, JIN XU², \\ LI-NA ZHANG ${ }^{2}$, WEN-HUA XING ${ }^{2}$, REN-JIE ZHUO ${ }^{2}$ and DUO LI ${ }^{1}$ \\ ${ }^{1}$ Department of Food Science and Nutrition, Zhejiang University, Hangzhou; ${ }^{2}$ Medical School, \\ Ningbo University, Ningbo; ${ }^{3}$ Clinical Laboratory, Lihuili Hospital, Ningbo, P.R. China
}

Received December 5, 2008; Accepted April 8, 2009

DOI: 10.3892/ijmm_00000212

\begin{abstract}
PI-103, the first synthetic multitargeted compound which simultaneously inhibits PI3K $\alpha$ and mammalian target of rapamycin (mTOR) shows high antitumor activity in glioma xenografts. In the present study, clear antitumor activity was observed with PI-103 treatment in two gefitinib-resistant nonsmall cell lung cancer (NSCLC) cell lines, A549 and H460, by simultaneously inhibiting p70s6k phosporylation and Akt phosphorylation in response to mTOR inhibition. In addition, H460 cells with activating mutations of PIK3CA were more sensitive to PI-103 than A549 cells with wild-type PIK3CA. PI-103 was found to inhibit growth by causing $\mathrm{G}_{0}-\mathrm{G}_{1}$ arrest in A549 and H460 cells. Western blotting showed that PI-103 induced down-regulation of cyclin D1 and E1 and simultaneously up-regulated p21 and p27, associated with arrest in the $\mathrm{G}_{0}-\mathrm{G}_{1}$ phase of the cell cycle. Furthermore, p53, the tumor suppressor which transcriptionally regulates $\mathrm{p} 21$, was also upregulated with PI-103 treatment. Collectively, our results suggest that multitargeted intervention is the most effective tumor therapy, and the cooperative blockade of PI3K $\alpha$ and mTOR with PI-103 shows promise for treating gefitinib-resistant NSCLC.
\end{abstract}

\section{Introduction}

Lung cancer is one of the most common cancers worldwide and only a minority of non-small cell lung cancer (NSCLC) patients are suitable for radical therapy including surgery, radiotherapy and chemotherapy as curative care (1). Therefore, the clinical development of targeted therapy agents appears to

Correspondence to: Dr Duo Li, Department of Food Science and Nutrition, Zhejiang University, 268 Kaixuan Road, Hangzhou, Zhejiang 310029, P.R. China

E-mail: duoli@zju.edu.cn

"Contributed equally

Key words: PI-103, PI3K $\alpha$, mammalian target of rapamycin, cell cycle, non-small cell lung cancer be on a promising course. Recently, gefitinib, a targeted agent which inhibits epidermal growth factor receptor (EGFR) tyrosine kinase, has entered into clinical trials for NSCLC. But only a small percent of NSCLC patients showed a response to it. In vitro studies demonstrated that gefitinib-resistant NSCLC cell lines show EGFR-independent activity of the PI3K/Akt/ mammalian target of rapamycin (mTOR) pathway, which resulted in the relative failure of first-generation single targeted therapies in lung cancer (2).

The PI3K/Akt/mTOR pathway is strongly implicated in human cancer. In cancer cells, commonly aberrant activation of the PI3K/Akt/mTOR pathway, which induces malignant transformation and chemoresistance, is due to genetic mutations $(3,4)$. The two most common mutations of the PI3K signaling pathway are somatic activating mutations of PIK3CA, the gene encoding the $\mathrm{p} 110 \alpha$ catalytic subunit of PI3K $\alpha$ and loss of the tumor suppressor PTEN $(5,6)$. Similarly, mTOR phosphorylation was found in $51 \%$ of NSCLC patient samples and in $74 \%$ of NSCLC cell lines (7). Moreover, previous studies show that functional crosstalk and specific feedback mechanisms exist among crucial signaling pathways (8). An example is that mTOR inhibition by rapamycin or its analogs triggers rapid and sustained activation of the PI3K/Akt survival pathway $(9,10)$. These results suggest that inhibiting EGFR alone is not sufficient for growth inhibition of all NSCLC cells, and highlight the usefulness of multipoint intervention in NSCLC, leading us to assess the rationale for a combined inhibition of both PI3K and mTOR targets in NSCLC cells.

PI-103 is a novel lipid kinase inhibitor of the PI3K signaling pathway with unique activity against glioma and human leukemic cell lines, owing to its ability to selectively block PI3K $\alpha$ and both mTOR complexes (11-14). Meanwhile, PI-103 was also highly effective and showed low cellular toxicity in glioma xenografts (15). However, the antitumor activity of PI-103 in gefitinib-resistant NSCLC remains elusive.

In the present study, two gefitinib-resistant NSCLC cell lines, the PIK3CA mutant $\mathrm{H} 460$ and the PIK3CA wild-type A549 were selected $(16,17)$. The antitumor activity of PI-103 in gefitinib-resistant NSCLC cell lines was assessed and the biomarkers as readouts of response to PI-103 were explored. Finally, we investigated the corresponding mechanisms of the antitumor effects of PI-103 in A549 and H460 cells. 


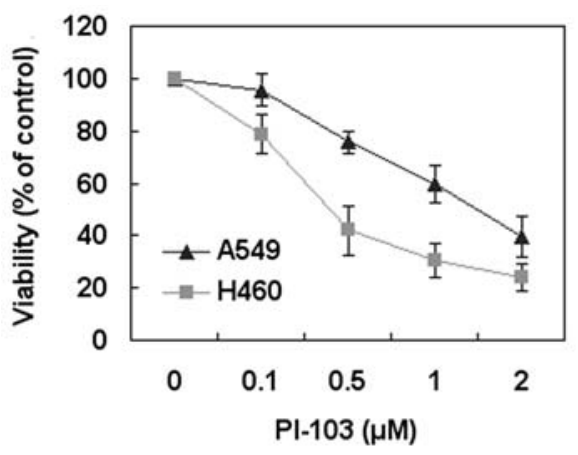

Figure 1. PI-103 inhibits A549 and H460 cell proliferation. A549 and H460 cells were treated with the indicated concentrations of PI-103 for $72 \mathrm{~h}$, viability was determined by MTT assay. Data represent means \pm SD of three independent observations performed in quintuplicate.

\section{Materials and methods}

Cells and culture. The NSCLC cell lines A549 and H460 were purchased from the cellbank of the Chinese Academy of Science. RPMI 1640 was used as culture media containing $10 \%$ heat-inactivated fetal calf serum (FCS). Cells were grown at $37^{\circ} \mathrm{C}$ in a humidified atmosphere with $5 \% \mathrm{CO}_{2}$. Cells from exponentially growing cultures were used in all experiments.

Chemicals and antibodies. PI-103 was purchased from Merck (Darmstadt, Germany). MTT [3-(4, 5)-dimethylthiazol-2-yl)-2, 5-diphenyltetrazolium bromide] was purchased from Sigma (St. Louis, MO). Coulter DNA prep reagents kit for cell cycle was purchased from Beckman Coulter (Fullerton, CA). Enhanced BCA Protein Assay Kit, enhanced chemiluminescence reagents and NP-40 lysis buffer were purchased from Beyotime Institute of Biotechnology (Jiangsu, China). Anti-phosphoAkt (Ser473), anti-Akt, anti-p70s6k from Santa Cruz Biotechnology (Santa Cruz, CA). Anti-phospho-Erk (Thr202/Tyr204), anti-Erk, anti-phospho-p70s6k (Tyr389), anti-cyclin E1 and D1, anti-p21, anti-p27 and anti-p53 from Cell Signaling Technology (Beverly, MA).

MTT assay for cell proliferation. For cellular survival assays, 3,000 cells were seeded into flat bottom 96-well plates. After $24 \mathrm{~h}$ of incubation, growth medium was replaced by fresh medium containing $10 \%$ FCS. Subsequently, various concentrations of the agents as indicated in the results were added and cells were incubated for an additional $72 \mathrm{~h}$. Next, MTT $(100 \mu \mathrm{g} /$ well) was added to each well and incubated for $4 \mathrm{~h}$ at $37^{\circ} \mathrm{C}$. The colored formazan product was then dissolved using $200 \mu \mathrm{l}$ of DMSO. Absorbance was determined at $492 \mathrm{~nm}$ using a microplate reader and absorbance values were expressed as the percentage of the untreated controls. All the assays were performed at least three times in quintuplicate.

Cell cycle analysis. To assess effects on the cell cycle, $5 \times 10^{5}$ H460 and A549 cells were seeded into 6-well plates. After $24 \mathrm{~h}$, cells were treated with various concentrations of the drugs as indicated in the results. After an additional $24 \mathrm{~h}$, cells were washed once with PBS and harvested. Subsequently, the harvested cells were incubated with reagents for cell cycle

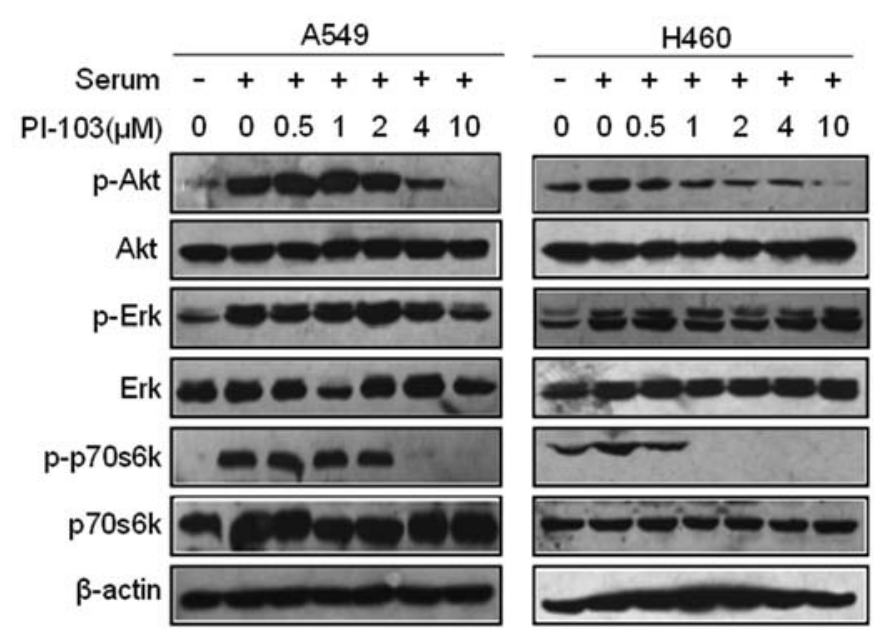

Figure 2. Effects of PI-103 on the phosphorylation of Akt, p70s6k, and Erk in A549 and H460 cells. A549 and H460 cells were cultured and subjected to serum starvation for $24 \mathrm{~h}$, and then treated with PI-103 at dosages shown for $1 \mathrm{~h}$. FCS $(10 \%)$ was added 15 min before harvest, and immunoblotting was performed as described in Materials and methods. Activation of Akt, p70s6k and Erk was determined using phosphor-specific antibodies.

from Beckman Coulter in the dark for $30 \mathrm{~min}$ and then cell cycle distribution was analyzed with a Becton Dickinson FACS Calibur flow cytometer.

Western blot analysis. H460 and A549 cells were treated with specified agents for $1 \mathrm{~h}$ at various concentrations in complete medium. The cells were lysed with ice-cold NP-40 lysis buffer containing protease inhibitor PMSF (1 nM) for $50 \mathrm{~min}$ on ice. Samples were centrifuged at $13,000 \mathrm{rpm}$ for $5 \mathrm{~min}$ at $4^{\circ} \mathrm{C}$. Protein context was determined using Bicinchoninic Acid assay and equal amounts of total protein were separated in $10 \%$ SDSpolyacrylamide gels, and then transferred to PVDF membranes. After blocking with 5\% nonfat dry milk for $4 \mathrm{~h}$ at room temperature, the membranes were incubated overnight with gentle shaking at $4^{\circ} \mathrm{C}$ with the primary antibodies as indicated in the results. The following day, the membranes were incubated with the appropriate horseradish peroxidase-conjugated secondary antibodies, and proteins were detected by enhanced chemiluminescence reagents.

\section{Results}

PI-103 induces growth inhibition in A549 and H460 cells. To determine the antiproliferative effects of PI-103 on the growth of human lung cancer cells, A549 and H460 cells were treated with PI-103 at dosages ranging from 0 to $2 \mu \mathrm{M}$ and MTT assay was conducted. As shown in Fig. 1, incubation of A549 cells with $2 \mu \mathrm{M}$ PI-103 for $72 \mathrm{~h}$ induced an $\sim 60 \%$ reduction in cell number. In contrast to A549 cells, H460 cells were highly sensitive to low-dose PI-103. Treatment of H460 cells with $0.5 \mu \mathrm{M}$ PI-103 for $72 \mathrm{~h}$ resulted in $\sim 60 \%$ inhibition. Results showed that exposure of A549 and H460 cells to PI-103 with the indicated concentrations for $72 \mathrm{~h}$ induced growth inhibition in a dose-dependent manner.

Effects of PI-103 on signaling pathways. To explore the mechanism by which PI-103 inhibits cell growth, the effects 


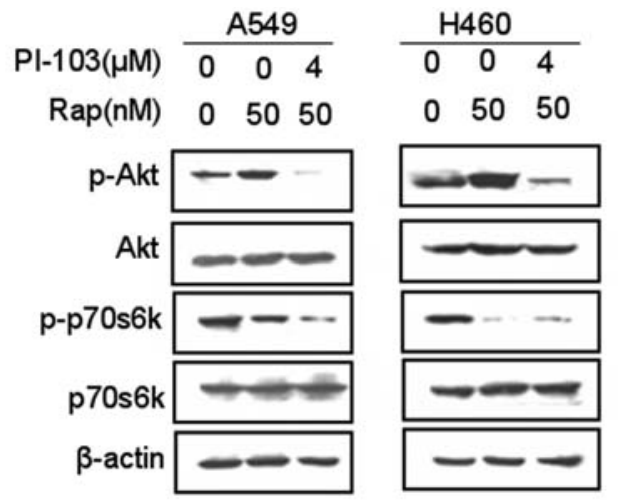

Figure 3. PI-103 blocks rapamycin-induced Akt phosphorylation. A549 and H460 cells were treated with rapamycin and PI-103 at dosages shown for $24 \mathrm{~h}$. Immunoblotting was performed as described in Materials and methods. Activation of Akt and p70s6k was determined using phosphor-specific antibodies.

of PI-103 on related intracellular signaling proteins were examined. H460 cells had high levels of p-Akt and p-p70s6k at baseline, correlating with activating mutations of PIK3CA in themselves. As expected, in A549 and H460 cells, p-p70s6k was impacted by PI-103 treatment, which changed in parallel with p-Akt. PI-103 in high doses completely and specifically abrogated basal and serum-induced phosphorylation of Akt and p70s6k in both cell types (Fig. 2). In H460 cells, phosphorylation of Akt was markedly affected by the treatment of PI-103 in low doses, but only modestly in A549 cells. In contrast, phosphorylation of Erk was hardly affected by PI-103. Similarly, Akt, p70s6k and Erk were not impacted by PI-103.

PI-103 reverses rapamycin-induced Akt overactivation. Treatment of A549 and H460 cells with rapamycin (50 nM) markedly inhibited phosphorylation of p70s6k (Fig. 3). Unfortunately, blockade of mTOR with rapamycin markedly induced increased Akt phosphorylation at Ser473. In both A549 and H460 cells, phosphorylation of Akt induced by inhibition of mTOR with rapamycin was significantly eradicated by PI-103 $(4 \mu \mathrm{M})$.

PI-103 blocks cell cycle progress in A549 and H460 cells. To explore whether growth inhibition of PI-103 is due to alterations in cell cycle progression, flow cytometry was used for cell cycle analysis. As shown in Fig. 4, treatment of A549 cells with PI-103 for $24 \mathrm{~h}$ showed an increase in $\mathrm{G}_{0}-\mathrm{G}_{1}$ DNA content. In addition, PI-103 $(1 \mu \mathrm{M})$ alone induced $\mathrm{G}_{0}-\mathrm{G}_{1}$ arrest more obviously in H460 than in A549 cells. PI-103 $(1 \mu \mathrm{M})$ induced an $20 \%$ increase in $\mathrm{G}_{0}-\mathrm{G}_{1}$ phase in $\mathrm{H} 460$ cells.

Effects of PI-103 on protein levels of important $G_{0}-G_{1}$ phase regulators. In A549 and $\mathrm{H} 460$ cells, $\mathrm{PI}-103$ treatment caused cell cycle arrest at $\mathrm{G}_{0}-\mathrm{G}_{1}$ phase (Fig. 4). To identify the pathway involved in the PI-103-induced cell cycle arrest in A549 and $\mathrm{H} 460$ cells, a series of studies were performed in which the expression of cyclins and cyclin-dependent kinase inhibitors that play important roles in $\mathrm{G}_{0}-\mathrm{G}_{1}$ arrest, was measured upon PI-103 treatment. In A549 and H460 cells, treatment with PI-103 led to significantly decreased cyclin

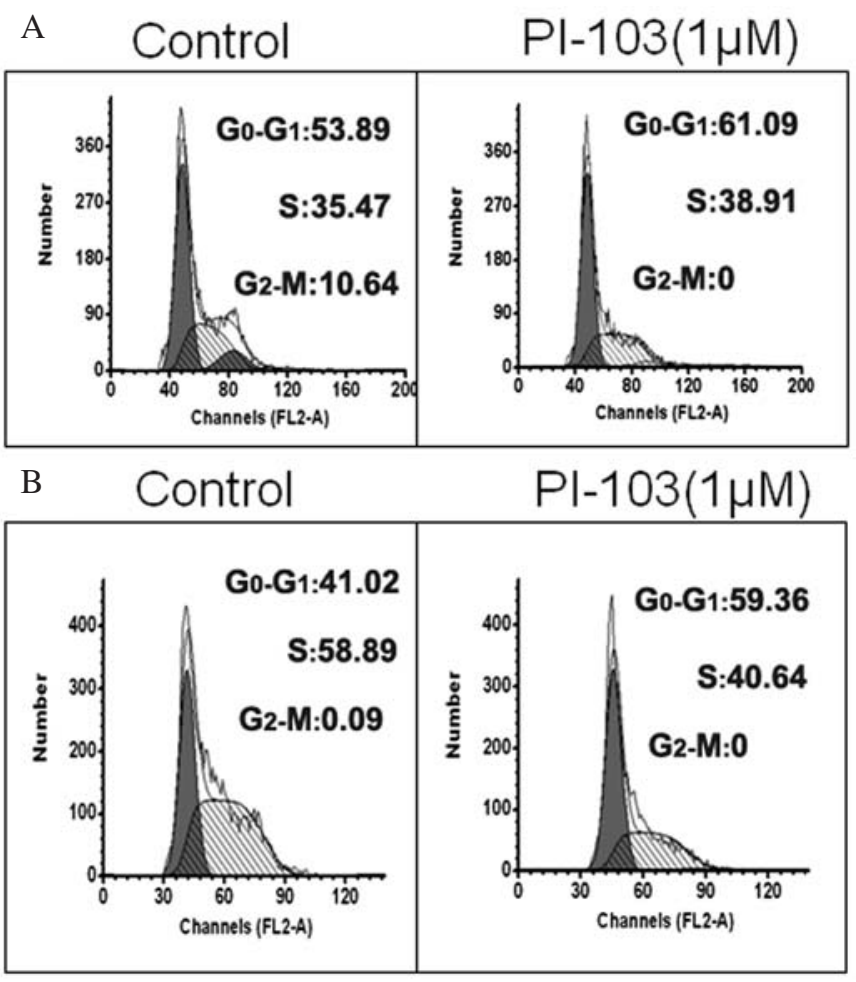

Figure 4. Effects of PI-103 on induction of cell cycle arrest in A549 and H460 cells. (A) A549 and (B) H460 cells were treated with PI-103 (1 $\mu \mathrm{M})$ for $24 \mathrm{~h}$. Cell cycle analysis was performed by flow cytometry after propidium iodine staining.

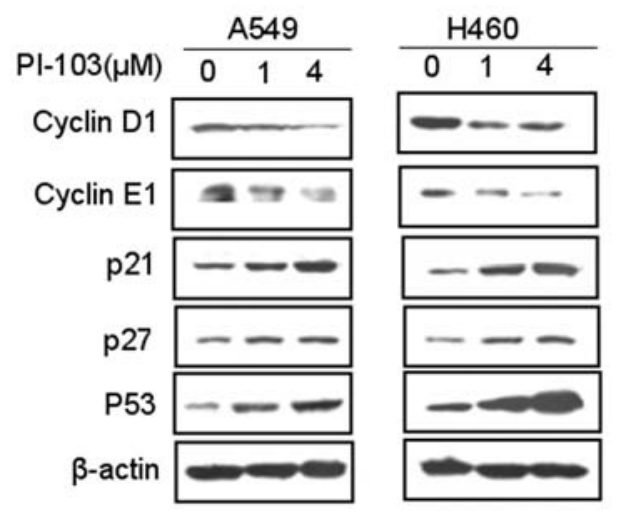

Figure 5. Effects of PI-103 on the expression of cell cycle regulatory proteins. A549 and H460 cells were treated with PI-103 at dosages shown for $24 \mathrm{~h}$. The indicated proteins were detected by Western blot analysis as described in Materials and methods.

D1 and E1 in a dose-dependent manner (Fig. 5). Upregulation of p21 and p27 was known to be involved in cell cycle arrest. Thus, the effects of PI-103 on p21 and p27 protein levels were examined. Expectedly, PI-103 treatment increased their expression in a dose-dependent manner, especially p21.p21 is a transcriptional target of p53 and is the major effector of the p53-mediated $\mathrm{G}_{1}$ cell cycle checkpoint (18). Thus, we addressed whether p21 induction is related to p53-dependent response in A549 and H460 cells with PI-103 treatment. As shown in Fig. 5, a marked increase in p53 protein level was observed following PI-103 treatment in A549 and H460 cells. 


\section{Discussion}

The PI3K/Akt/mTOR signaling pathway was recently identified as a potential target for therapeutic intervention in NSCLC. The aberrant activation of the PI3K/Akt/mTOR pathway correlates with a resistance to gefitinib and a poor prognosis in NSCLC patients (2). This suggests that in gefitinibresistant NSCLC cell lines, intervention at multiple points in signaling cascades is necessary to most effectively inhibit cell growth and survival. PI-103, the first synthetic multitargeted compound, which specifically and simultaneously inhibits $\mathrm{PI} 3 \mathrm{~K} \alpha$ and both mTOR complexes shows its potential for the treatment of malignant brain tumors (gliomas) (15). Therefore, PI-103 may represent an emerging paradigm for the treatment of gefitinib-resistant NSCLC.

In this study, immunoblotting experiments verified that treatment of A549 and H460 cells with PI-103 not only inhibited Akt phosphorylation but also blocked p70s6k phosphorylation, but hardly impacted phosphorylation of Erk, indicating that PI-103 specifically targets PI3K $\alpha$ and mTOR. In contrast to PI-103, rapamycin, the inhibitor of mTOR significantly suppressed phosphorylation of p70s6k and simultaneously induced increased activation of Akt (Fig. 3), a phenomenon previously reported by others $(10,19)$. The potential feedback inhibition whereby downregulation of mTOR-S6K signaling by rapamycin activates the PI3K/Akt pathway through IRS or IGF-1R-dependent mechanism may limit the efficacy of rapamycin as a single agent (20). Importantly, treatment of A549 and H460 cells with PI-103 not only inhibited p70s6k phosphorylation, but also blocked Akt phosphorylation in response to mTOR inhibition (Fig. 3). Flow cytometric analysis and MTT assay experiments further confirmed the efficacy of PI-103 by concomitantly inhibiting both PI3K $\alpha$ and mTOR in A549 and H460 cells.

A series of studies demonstrated an association between cell cycle regulation and tumors, and the blockade of the cell cycle progression has become an appropriate target for tumor therapy (21-23). Cell cycle analysis showed that PI-103 induced cell cycle arrest in $\mathrm{G}_{0}-\mathrm{G}_{1}$ phase in A549 and $\mathrm{H} 460$ cells. As is known, the cell cycle is tightly regulated by cell cycle regulatory proteins (cyclins, CDKs, and CDK inhibitors) (24). Cyclin D/CDK and E/CDK complexes are required for initiation of cell division in $\mathrm{G}_{1}$ phase and for progression into $\mathrm{S}$ phase respectively (25). p21 and p27, the inhibitors of CDKs, are known to be required for cell cycle arrest at $G_{0}-G_{1}$ phase. Moreover, the upregulation of p21 is reported to be modulated by p53 (26). In our results, we found that cyclin D1 and E1 were inhibited in a dose-dependent manner in A549 and H460 cells with PI-103 treatment. In contrast, p21, p27 and p53 were strikingly induced with PI-103 treatment, suggesting that PI-103-induced growth arrest of A549 and H460 cells is associated with a sequential regulation of cyclins and cyclindependent kinase inhibitors (CKIs), which modulates the cell cycle machinery and inhibits the $\mathrm{G}_{1}-\mathrm{S}$ phase progression. Our data also show that PI-103-induced $\mathrm{G}_{0}-\mathrm{G}_{1}$ arrest is dependent on p53 in A549 and $\mathrm{H} 460$ cells.

Although H460 cells possess higher basal protein levels of PTEN than A549 cells (16), the high activity of the PI3K/ Akt/mTOR pathway due to activating mutations of PIK3CA contributes to high antitumor activity of PI-103 in H460 cells.
These data suggest that signaling transduction inhibitors are effective primarily in cases where the targeted kinase has undergone mutational activation. Our results further support the phenomenon of oncogene addiction in which the apparent dependency of cancer cells on individual oncogenes to survive renders them susceptible to targeted inhibitors (27).

Tumors depend on complex signaling networks to promote malignant transformation and dynamically adapt to stress. Blocking only one of these pathways allows others to act as salvage or escape mechanisms for cancer cells. Thus, it is becoming increasingly apparent that the most effective targeted cancer therapies owe to their concomitant inhibition of multiple targets. The high efficacy of multitargeted intervention was previously described for NSCLC, human melanoma and other tumor types (28-30). In the present study, the dual inhibitor PI-103 indeed showed obvious antitumor activity in gefitinibresistant NSCLC cell lines as seen in glioma and other tumor cell lines, further reinforcing the importance of multitargeted therapeutics $(11,31,32)$. Additionally, we identified that activating mutations of $P I K 3 C A$ were a crucial determinant of response to low-dose PI-103. Based on the efficacy of PI-103 in gefitinib-resistant NSCLC cell lines, PI-103 is hypothesized to be another potential targeted inhibitor following EGFR inhibitors gefitinib, erlotinib and ZD6474 in NSCLC treatment. Our data above suggest that concomitant inhibition of PI $3 \mathrm{~K} \alpha$ and mTOR with PI-103 is a promising therapeutic strategy for treating gefitinib-resistant NSCLC. Although the efficacy and tolerability of PI-103 has to be examined in preclinical models, our work is relevant to the clinic of NSCLC, as many specific signal transduction inhibitors are being developed for clinical use.

\section{Acknowledgements}

We are grateful to Xiao-Lin Lu for the cell culture; Li Wang, Zhen-Zhen Cai and Gao-Feng Yuan for useful discussion.

\section{References}

1. Jemal A, Murray T, Samuels A, Ghafoor A, Ward E and Thun MJ: Cancer statistics, 2003. CA Cancer J Clin 53: 5-26, 2003.

2. Janmaat ML, Kruyt FAE, Rodriguez JA and Giaccone G: Response to epidermal growth factor receptor inhibitors in nonsmall cell lung cancer cells: limited antiproliferative effects and absence of apoptosis associated with persistent activity of extracellular signal-regulated kinase or Akt kinase pathways. Clin Cancer Res 9: 2316-2326, 2003.

3. Haluska FG, Tsao H, Wu H, Haluska FS, Lazar A and Goel V: Genetic alterations in signaling pathways in melanoma. Clin Cancer Res 12: 2301-2307, 2006.

4. Nicholson KM and Anderson NG: The protein kinase B/Akt signalling pathway in human malignancy. Cell Signal 14: 381-395, 2002.

5. Engelman JA: The role of phosphoinositide 3-kinase pathway inhibitors in the treatment of lung cancer. Clin Cancer Res 13: 4637-4640, 2007.

6. Morrow CJ, Gray A and Dive C: Comparison of phosphatidylinositol-3-kinase signalling within a panel of human colorectal cancer cell lines with mutant or wild-type PIK3CA. FEBS Lett 579: 5123-5128, 2005.

7. Balsara BR, Pei J, Mitsuuchi Y, Page R, Klein-Szanto A, Wang H, Unger M and Testa JR: Frequent activation of AKT in nonsmall cell lung carcinomas and preneoplastic bronchial lesions. Carcinogenesis 25: 2053-2059, 2004.

8. Kim GE, Kim YB, Cho NH, Chung HC, Pyo HR, Lee JD, Park TK, Koom WS, Chun M and Suh CO: Synchronous coexpression of epidermal growth factor receptor and cyclooxygenase- 2 in carcinomas of the uterine cervix: A potential predictor of poor survival. Clin Cancer Res 10: 1366-1374, 2004. 
9. Chiang GG and Abraham RT: Targeting the mTOR signaling network in cancer. Trends Mol Med 13: 433-442, 2007.

10. Sun SY, Rosenberg LM, Wang X, Zhou Z, Yue P, Fu H and Khuri FR: Activation of Akt and eIF4E survival pathways by rapamycin-mediated mammalian target of rapamycin inhibitioned. Cancer Res 65: 7052-7058, 2005.

11. Fan QW, Cheng CK, Nicolaides TP, Hackett CS, Knight ZA, Shokat KM and Weiss WA: A dual phosphoinositide-3kinase $\{$ alpha $\} / \mathrm{mTOR}$ inhibitor cooperates with blockade of epidermal growth factor receptor in PTEN-mutant glioma. Cancer Res 67: 7960-7965, 2007.

12. Knight ZA, Gonzalez B, Feldman ME, Zunder ER, et al: A pharmacological map of the PI3-K family defines a role for p110[alpha] in insulin signaling. Cell 125: 733-747, 2006.

13. Kojima K, Shimanuki M, Shikamil M, et al: The dual PI3 kinase $\alpha /$ mTOR inhibitor PI-103 prevents $\mathrm{p} 53$ induction by Mdm2 inhibition but enhances p53-mediated mitochondrial apoptosis in p53 wild-type AML. Leukemia 22: 1728-1736, 2008.

14. Park S, Chapuis N, Bardet V, et al: PI-103, a dual inhibitor of Class IA phosphatidylinositide 3-kinase and mTOR, has antileukemic activity in AML. Leukemia 22: 1698-1706, 2008.

15. Fan QW, Knight ZA, Goldenberg DD, Yu W, Mostov KE, Stokoe D, Shokat KM and Weiss WA: A dual PI3 kinase/mTOR inhibitor reveals emergent efficacy in glioma. Cancer Cell 9: 341-349, 2006

16. Janmaat ML, Rodriguez JA, Gallegos-Ruiz M, Kruyt FA and Giaccone G: Enhanced cytotoxicity induced by gefitinib and specific inhibitors of the Ras or phosphatidyl inositol-3 kinase pathways in non-small cell lung cancer cells. Int J Cancer 118: 209-214, 2006.

17. Whyte DB and Holbeck SL: Correlation of PIK3Ca mutations with gene expression and drug sensitivity in NCI-60 cell lines. Biochem and Biophys Res Commun 340: 469-475, 2006.

18. Sherr CJ and Roberts JM: Inhibitors of mammalian G1 cyclindependent kinases. Genes Dev 9: 1149-1163, 1995.

19. O'Reilly KE, Rojo F, She QB, et al: mTOR inhibition induces upstream receptor tyrosine kinase signaling and activates Akt Cancer Res 66: 1500-1508, 2006.

20. Wan X, Harkavy B, Shen N, Grohar P and Helman LJ: Rapamycin induces feedback activation of Akt signaling through an IGF-1Rdependent mechanism. Oncogene 26: 1932-1940, 2006.

21. Jacks T and Weinberg RA: Cell-cycle control and its watchman. Nature 381: 643-644, 1996
22. Shukla S and Gupta S: Molecular mechanisms for apigenininduced cell-cycle arrest and apoptosis of hormone refractory human prostate carcinoma DU145 cells. Mol Carcinog 39: 114-126, 2004.

23. Singh RP, Agarwal C and Agarwal R: Inositol hexaphosphate inhibits growth, and induces G1 arrest and apoptotic death of prostate carcinoma DU145 cells: modulation of CDKI-CDKcyclin and pRb-related protein-E2F complexes. Carcinogenesis 24: 555-563, 2003.

24. Li W, Sanki A, Karim R, Thompson J, Soon Lee C, Zhuang L, McCarthy S and Scolyer R: The role of cell cycle regulatory proteins in the pathogenesis of melanoma. Pathology 38: 287-301, 2006.

25. Galea CA, Wang Y, Sivakolundu SG and Kriwacki RW: Regulation of cell division by intrinsically unstructured proteins: intrinsic flexibility, modularity, and signaling conduits. Biochemistry 47: 7598-7609, 2008.

26. Liu G and Lozano G: p21 stability: Linking chaperones to a cell cycle checkpoint. Cancer Cell 7: 113-114, 2005.

27. Sharma SV, Gajowniczek P, Way IP, et al: A common signaling cascade may underlie 'addiction' to the Src, BCR-ABL, and EGF receptor oncogenes. Cancer Cell 10: 425-435, 2006.

28. Buck E, Eyzaguirre A, Brown E, et al: Rapamycin synergizes with the epidermal growth factor receptor inhibitor erlotinib in non-small-cell lung, pancreatic, colon, and breast tumors. Mol Cancer Ther 5: 2676-2684, 2006.

29. Chun PY, Feng FY, Scheurer AM, Davis MA, Lawrence TS and Nyati MK: Synergistic effects of gemcitabine and gefitinib in the treatment of head and neck carcinomaed. Cancer Res 66: 981-988, 2006.

30. Nakamura H, Takamori S, Fujii T, Ono M, Yamana H, Kuwano M and Shirouzu K: Cooperative cell-growth inhibition by combination treatment with ZD1839 (Iressa) and trastuzumab (Herceptin) in non-small-cell lung cancer. Cancer Lett 230: 33-46, 2005.

31. Chen JS, Zhou LJ, Entin-Meer M, et al: Characterization of structurally distinct, isoform-selective phosphoinositide 3'-kinase inhibitors in combination with radiation in the treatment of glioblastoma. Mol Cancer Ther 7: 841-850, 2008.

32. Prevo R, Deutsch E, Sampson O, Diplexcito J, Cengel K, Harper J, O'Neill P, Mckenna WG, Patel S and Bernhard EJ: Class I PI3 kinase inhibition by the pyridinylfuranopyrimidine inhibitor PI-103 enhances tumor radiosensitivity. Cancer Res 68: 5915-5923, 2008. 